\title{
Characteristics and prognosis of coexisting adnexa malignancy with endometrial cancer: a single institution review of 51 cases
}

\author{
Lu Chen $\cdot$ Qiang Zhao $\cdot$ Xiaojuan Lv
}

Received: 30 April 2010 / Accepted: 15 June 2010 / Published online: 29 June 2010

(C) The Author(s) 2010. This article is published with open access at Springerlink.com

\begin{abstract}
Objective To explore and compare the differences in the clinicopathological characteristics and prognosis of synchronous primary endometrial and ovarian cancers with primary endometrial cancer metastatic to adnexa.

Materials and methods Between January 1997 and December 2009, 51 cases with endometrial cancer simultaneously with adnexa malignancy were identified. Among them, there were 18 cases with synchronous primary cancers of the endometrium and ovary (Group A) and 33 cases with primary endometrial cancer metastatic to the adnexa (Group B). Clinical and pathologic information was obtained from medical records. Parametric methods were used to compare clinical and pathologic features. KaplanMeier survival analysis was performed and compared using log-rank test.

Results The mean age at diagnosis of the disease was $56.6 \pm 10.8$ years (range 34-76 years) in Group A and $53.1 \pm 9.5$ years (range $37-76$ years) in Group B. The two groups' distribution of preoperative image findings, size of endometrial lesion, myometrial invasion, unilateral or bilateral, cervix invasion, and postoperative radiation existed significant differences. With a mean follow-up time of
\end{abstract}

L. Chen and X. Lv contributed equally to this work.

L. Chen $\cdot$ Q. Zhao $(\bowtie) \cdot X$. Lv

Department of Gynecology Oncology,

Zhejiang Provincial Cancer Hospital,

38 Guangji Road, Hangzhou, People's Republic of China

e-mail: zq1990888@126.com

L. Chen

e-mail: zq1990876@126.com

X. Lv

e-mail: 1vxiaojuan1984@163.com
$4.3 \pm 3.4$ years (range 2-11 years), 5-year overall survival (OS) was 75 and 56\% in Groups A and B, respectively $(p=0.034)$. The univariate analysis showed only postoperative radiation and synchronous tumors were independent factors which affected OS $(p=0.015 ; p=0.034)$ and progression-free survival (PFS) $(p=0.015 ; p=0.036)$, respectively. Not any feature was revealed by multivariate analysis as independent prognostic factors.

Conclusion Our results showed that OS and PFS of synchronous primary ovarian cancer in patients with endometrial cancer is better than those with ovarian metastasis patients. Pre- and intra-operative, intensive and careful assessment, and strict and continuous postoperative surveillance should pay attention to the endometrial cancer patients who preserved ovary for having possibility of coexisting occult ovarian lesions.

Keywords Endometrial neoplasm - Adnexa neoplasm . Synchronous primary cancer $\cdot$ Prognosis

\section{Introduction}

It has been identified that primary cancer can occur in the female reproductive organs simultaneously, especially with endometrial and ovarian cancers. Endometrial and adnexa malignancy may coexist in approximately $5 \%$ of all patients with endometrial cancer and $10 \%$ of those with ovarian cancer [1-4]. Patients with disease of both the endometrium and the ovary can be classified into three groups: (1) synchronous primary cancers of the endometrium and ovary, (2) endometrial cancer with metastasis to the adnexa, and (3) ovarian cancer with metastasis to the endometrial. Ulbright and Roth [5] proposed a set of histological criteria to distinguish the first two groups in 1985 . 
Then Scully et al. [1] described a similar but more extensive list of clinical pathologic features used to differentiate all the three groups. But there are still no absolute surgical or histological criteria.

The aim of the present retrospective study was to assess the clinical characteristics, pathologic patterns, and survival experience of the 51 endometrial cancer patients simultaneously with adnexa malignancy.

\section{Materials and methods}

Between January 1997 and December 2009, the Department of Gynecology Oncology of Zhejiang Provincial Cancer Hospital in China received and treated 818 patients with primary endometrial cancer. Patients who received preoperative radiation therapy or did not undergo surgery were excluded. Patients' medical history, pathologic reports, and treatment records were collected. There were 51 cases of primary endometrial cancer coexisting with adnexa malignancies: among them, 18 cases are synchronous primary cancers of the endometrium and ovary, and 33 cases are endometrial cancer with metastasis to the adnexa. Clinical information regarding age, preoperative image examination and serum CA125 level, postoperative chemotherapy, and/ or radiation was gathered. Histologic and pathologic information was collected, including tumor size, histological type, pathologic grade, peritoneal fluid cytology, invasive depth of myometrium, uterine serosal involvement, lymphvascular space invasion (LVSI), cervical involvement, and adnexa involvement.

All pathologic specimens were reviewed by our pathologists according to the criteria described by Scully et al. The pathologic criteria for synchronous primary cancers of the endometrium and ovary were as follows: (1) histological dissimilarity of tumors; (2) no or only superficial myometrial invasion of endometrial tumor; (3) no vascular space invasion of endometrial tumor; (4) atypical endometrial hyperplasia additionally present; (5) absence of other evidence of spread of endometrial tumor; (6) ovarian tumor unilateral (80-90\% of cases); (7) ovarian tumors located mainly in parenchyma; (8) no vascular space invasion, surface implants, or predominant hilar location in ovary; (9) absence of other evidence of spread of ovarian tumor; and (10) ovary endometriosis present.

Patients were divided into two groups according to the histology of endometrial and ovarian. Group A meant patients with synchronous endometrial and ovarian cancers. Group B meant patients with endometrial cancers with metastasis to adnexa. All patients' stages were reviewed and updated, based on the endometrial and ovarian cancer staging criteria set by the International Federation of Gynecology and Obstetrics (FIGO) [6].
Statistical analyses were performed using SPSS version 15.0 (SPSS Inc., Chicago, IL, USA). Pearson $\chi^{2}$ or Fisher's exact test was carried out for analyzing the categorical variables with respect to the effects of clinical and pathological risk factors on the coexisting adnexa malignancy rate. Associations between prognostic variables and survival were compared using the log-rank and $\chi^{2}$ tests, with a $p$ value $<0.05$ considered significant. Multivariate analysis was performed using Cox proportional hazards method. We also analyzed follow-up results including progression-free and overall survival (OS) of the two group patients, and comprised survival curves performed using the KaplanMeier method.

\section{Results}

The median age at diagnosis of the disease was $56.6 \pm 10.8$ years (range 34-76 years) in Group A and $53.1 \pm 9.5$ years (range 37-76 years) in Group B ( $p=$ 0.727). All patients underwent surgical staging with total or radical abdominal hysterectomy $(\mathrm{TH})$, bilateral salpingooophorectomy (BSO), and selective or systemic bilateral pelvic or/and para-aortic lymphadenectomy, according to the NCCN Clinical Practice Guidelines in Oncology-Uterine Neoplasms (2008). Patients with positive cervical biopsy result or gross cervical involvement received radical hysterectomy, BSO, cytology, and pelvic and para-aortic lymph node dissection. TH/BSO, cytology, maximal debulking, omentectomy, and pelvic and para-aortic lymph node dissection were carried out when patients had been found intra-abdominal lesions including ascites, omentum, nodal, and peritoneal disease. Before abdominal operation started, each patient received peritoneal washing for cytological examination. Pelvic and para-aortic lymph nodes were dissected in 88.3 and $52.9 \%$ of patients, respectively. 29 cases (56.9\%) received omentum resection. The histology coincidence rate of endometrium and ovary was $72.2 \%$, while 5 cases $(27.78 \%)$ had different pathologic types in Group A, including endometrioid carcinoma, papillary adenocarcinoma, and serous papillary adenocarcinoma. In Group B, 12 cases $(36.36 \%)$ were unilateral salpingo metastasis and 7 cases $(21.21 \%)$ with bilateral salpingo metastasis. Thirtyeight patients $(74.5 \%)$ underwent postoperative therapy. Among them, 8 (15.7\%) had radiation alone; 24 (47.1\%) had chemotherapy alone; and $4(7.8 \%)$ had both radiation and chemotherapy after operation.

Table 1 showed the clinical and pathological features and the differences of the constituent ratio of these two groups. The distribution of features was similar between two groups, including age, preoperative serum CA125 level, peritoneal washing, histology, grade, lymph-vascular invasion, pelvic lymph nodes, and para-aortic lymph nodes. 
Table 1 Clinical and pathological characteristics

\begin{tabular}{|c|c|c|c|}
\hline Variables & $\begin{array}{l}\text { Group A }(\%) \\
(n=18)\end{array}$ & $\begin{array}{l}\text { Group B }(\%) \\
(n=33)\end{array}$ & $p$ value \\
\hline Mean age (years) & $56.6 \pm 10.8$ & $53.1 \pm 9.5$ & 0.727 \\
\hline$>45$ & $14(77.78)$ & $27(81.82)$ & \\
\hline$\leq 45$ & $4(22.22)$ & $6(18.18)$ & \\
\hline Serum CA125 (U/ml) & & & 0.696 \\
\hline$\leq 35$ & $2(11.11)$ & $6(18.18)$ & \\
\hline$>35$ & $16(88.89)$ & $27(81.82)$ & \\
\hline Preoperative image findings & & & 0.042 \\
\hline Yes & $11(61.11)$ & $10(30.30)$ & \\
\hline No & $7(38.89)$ & $23(69.70)$ & \\
\hline Peritoneal cytology & & & 1.000 \\
\hline Negative & $15(83.33)$ & $27(81.82)$ & \\
\hline Positive & $3(16.67)$ & $6(18.18)$ & \\
\hline Size of endometrial lesion & & & 0.013 \\
\hline$\leq 2 \mathrm{~cm}$ & $16(88.89)$ & $17(51.52)$ & \\
\hline$>2 \mathrm{~cm}$ & $2(11.11)$ & $16(48.48)$ & \\
\hline Endometrial histology & & & 0.732 \\
\hline Endometrioid type & $13(72.22)$ & $26(78.79)$ & \\
\hline Non-endometrioid type & $5(27.78)$ & $7(21.21)$ & \\
\hline Endometrial tumor grade & & & 0.055 \\
\hline 1 & $9(50.00)$ & $7(21.21)$ & \\
\hline 2 & $6(33.33)$ & $11(33.33)$ & \\
\hline 3 & $3(16.67)$ & $15(45.46)$ & \\
\hline Myometrial invasion & & & 0.002 \\
\hline Limited to endometrium & $5(27.78)$ & $1(3.03)$ & \\
\hline$<1 / 2$ & $11(61.11)$ & $14(42.42)$ & \\
\hline$\geq 1 / 2$ & $2(11.11)$ & $18(54.55)$ & \\
\hline Ovary malignancy involvement & & & 0.015 \\
\hline Unilateral & $13(72.22)$ & $13(39.39)$ & \\
\hline Bilateral & $5(27.78)$ & $9(27.27)$ & \\
\hline Cervix invasion & & & 0.004 \\
\hline No & $15(83.33)$ & $12(36.36)$ & \\
\hline Yes & $3(16.67)$ & $21(63.64)$ & \\
\hline LVSI & & & 0.134 \\
\hline No & $17(94.44)$ & $25(75.76)$ & \\
\hline Yes & $1(5.56)$ & $8(24.24)$ & \\
\hline Pelvic lymph nodes & & & 0.136 \\
\hline Negative & $11(61.11)$ & $22(66.67)$ & \\
\hline Positive & $1(5.56)$ & $11(33.33)$ & \\
\hline Not available & $6(33.33)$ & 0 & \\
\hline Para-aortic lymph nodes & & & 0.631 \\
\hline Negative & $4(22.22)$ & $12(36.36)$ & \\
\hline Positive & $1(5.56)$ & $7(21.21)$ & \\
\hline Not available & $13(72.22)$ & $14(42.42)$ & \\
\hline Postoperative radiation & & & 0.004 \\
\hline No & $18(100)$ & $21(63.64)$ & \\
\hline Yes & $0(0)$ & $12(36.36)$ & \\
\hline Postoperative chemotherapy & & & 0.003 \\
\hline No & $3(16.67)$ & $20(60.61)$ & \\
\hline Yes & $15(83.33)$ & 13 (39.39) & \\
\hline
\end{tabular}

But the two groups' distribution of preoperative image findings, size of endometrial lesion, myometrial invasion, unilateral or bilateral, cervix invasion, and postoperative radiation existed significant differences.

With a mean follow-up time of $4.3 \pm 3.4$ years (range 2-11 years), 3- and 5-year OS was 82 and 75\%, respectively, in Group A. In Group B, 3- and 5-year OS was 65 and 56\%, respectively. The difference in OS between the two groups was significant $(Z=4.496, p=0.034$; Fig. 1$)$. Mean progression-free survival (PFS) was also significantly longer in Group A ( $3.9 \pm 3.2$ years) compared to Group B (2.8 \pm 2.1 years, $Z=6.552, p=0.010$; Fig. 2 ).

The log-rank univariate analysis of the two groups, only postoperative radiation $\left(\chi^{2}=5.873, p=0.015 ; \chi^{2}=5.887\right.$, $p=0.015)$ and synchronous tumors $\left(\chi^{2}=4.496, p=0.034\right.$; $\chi^{2}=4.387, p=0.036$ ), showed that these two parameters independently affected OS and PFS. There was no feature revealed by multivariate analysis (Cox regression) as an independent prognostic factor.

\section{Discussion}

It has a low incidence of endometrial cancers coexisting with adnexa tumors. Still there are no standard criteria for diagnosis and treatment of this kind of patients. Synchronous primary cancers of the endometrium and ovary occur in approximately $10 \%$ of all women with ovarian cancer and 5\% of all women with endometrial cancer [7-9]. The present study demonstrated that the overall rate of coexisting adnexa malignance in endometrial cancer patients was $6.23 \%$. Among this, $2.20 \%$ patients were synchronous primary cancers of endometrium and ovary, while 4.34\% patients were endometrial cancer with adnexa metastasis. It may be resulted from various human race and different area.

It had not found any significant difference from the mean age of these two groups. The common symptoms were lack of speciality, and similar to endometrial or ovarian cancer alone, including abnormal vaginal bleeding, ascites, abdominal pain, pelvic mass, and abdominal bloating. The early detection of ovarian cancer was likely due to early symptom related to concurrent endometrial cancer [1]. Most of synchronous patients had clinical characteristics, including young age, premenopausal, obesity, and nulliparity. Patients with concordant endometrial tumors had a favorable prognosis [2]. Similar results were obtained in our study; synchronous cancer patients usually had earlier stage, unilateral mass, smaller endometrial lesions, and with more superficial invasion to endometrium. Therefore, these patients were easily detected and received early treatment, and had better prognosis than those with metastasis group. Chemotherapy was commonly used for Group A by 


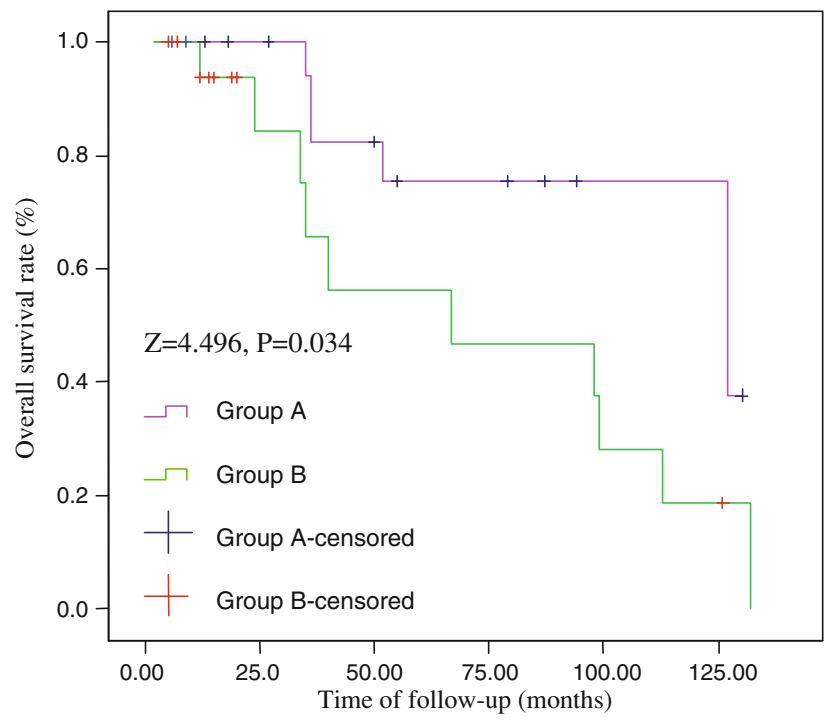

Fig. 1 Kaplan-Meier chart for overall survival (OS). Survival curve showed that OS of Group A was superior to that of Group B. "Censored" means cases without endpoint event at the end of follow-up

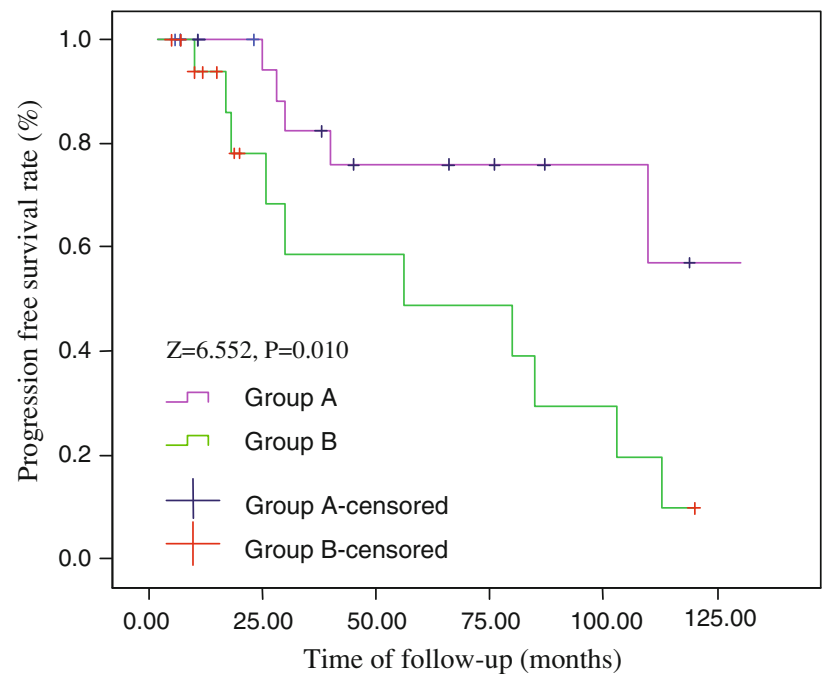

Fig. 2 Kaplan-Meier chart for progression-free survival (PFS). PFS curve showed that PFS of Group A was superior to that of Group B. "Censored" means cases without endpoint event at the end of follow-up

most of the doctors due to two sites of lesion. This retrospective study cannot further assess the role of chemotherapy and radiation because of the variety of adjuvant regiment administered. Up to now, there is no standard method to the postoperative adjuvant therapies for patients with synchronous endometrial and ovarian cancers. Because of small number of cases, the influence of the postoperative adjuvant therapy needs to be further investigated.

The GOG study found that 74 patients with simultaneously detected endometrial and ovarian cancers had an overall good prognosis with a 5 -year survival of $85.9 \%$ and 10-year survival of $80.3 \%$ [3]. In our cohort, it was obvious that the survival rate of Group A was better than that of Group B. We found that patients with synchronous primary cancers had a better survival than those with metastasis. This result was similar to data reported in the literature [2, 7]. Prognostic correlation factors of synchronous endometrial and ovarian cancers, including grade, early stage, and deep myometrial invasion [10,11], are frequently seen. Univariate analysis confirmed that synchronous tumors and postoperative radiation were two independent prognostic factors of survival. But there was no statistically significant difference in prognosis by multivariate analysis (Cox regression).

The incidence of ovarian metastasis in women with clinical stage I endometrial cancer has been reported, by most studies, to be approximately 5\%. This rate of ovarian metastasis has provided the basis for practice of removing both ovaries at the time of surgery, even in young patients. Gemer et al. [12] reported that the ovarian metastasis is microscopic in $18-70 \%$ of the endometrial carcinoma. Preoperative imaging studies or macroscopic intra-operative appearance cannot be relied on to exclude their presence [12]. Our data also showed that ovary metastasis of endometrial cancer was not easy to be detected by preoperative imaging detections.

It should be noted that ten cases (19.61\%) were aged less than 45 years when they were diagnosed in our study. Walsh et al. [4] reported among 102 young women who underwent hysterectomy for endometrial cancer: 26 (25\%) were found to have coexisting epithelial ovarian tumors, 23 were classified as synchronous primaries, and 3 as metastasis. Careful pre- and intra-operative assessment of adnexa is mandatory in young women with endometrial cancer [4]. However, Wright et al. [13] reported that ovarian preservation in premenopausal women with early-stage endometrial cancer might be safe and not associated with an increase in cancer-related mortality. Despite the potential for occult ovarian tumors in women undergoing ovarian preservation, survival was not compromised in their series. The risk should be taken into consideration when preserving the ovary in the treatment for young patients [14-18]. Macroscopic appearance of the ovary should not be relied on as an only basis. Kotera et al. [19] reported that it was valuable and feasible for early stage (stage IA) epithelial ovarian cancer patient who underwent opposite ovary wedge resection. One can check whether it exists opposite ovary metastases through frozen section; on the other hand, it can preserve endocrine function. Therefore, careful intra-operative evaluation, wedge-shaped resection of ovary and frozen section seems necessary before preserving the ovary. Careful oncologic, psychotherapeutic, genetic, and reproductive counseling is advised before offering a non-standard treatment strategy to young endometrial cancer patients. 
Long-term risks and benefits of ovarian preservation should be forward discussed [20]. The pathologists and clinical oncologists should pay more attention to the problem of making diagnosis and individual treatment [21].

Patients with synchronous ovarian and endometrial cancers may represent cases of a single primary tumor with metastasis (SPM) or dual primary tumors (DP). Ramus et al. [22] showed that genetic analysis may represent a powerful tool for use in clinical practice for distinguishing between SPM and DP in patients with synchronous ovarian/endometrial cancer and predicting disease outcome. The data also suggested a hitherto uncharacterized level of heterogeneity in these cases, which, if accurately defined, could lead to improved treatment and survival. In the future, this might lead to improved clinical management and outcome for the disease.

\section{Conclusion}

Our results agree with recent reports which found pathologic feature and outcome of patients with synchronous endometrial and ovarian cancers. Synchronous cancer patients usually have early stage, unilateral mass, small endometrial lesions, and with more superficial invasion to endometrium. Despite the retrospective design and limited cases of this study, our results suggest that endometrial cancer patients with synchronous primary cancer have a better survival than those with ovarian metastasis group. Intensive and careful assessment is required despite the incidence of the occult ovarian lesion with young endometrial cancer patients is uncommon. Endometrial patients who preserved ovary need to have strict and continued postoperative surveillance to the retained adnexa.

Acknowledgments We sincerely thank Drs. Wenyong Sun and Wei $\mathrm{Wu}$, who are from Department of Pathology, Zhejiang Provincial Cancer Hospital, for their major input into this study.

Conflict of interest statement The authors declare that they have no conflict of interest. They also indicate that they had no financial relationship with the organization that sponsored the research. They had full control of all primary data, and they agree to allow the journal to review their data if required.

Open Access This article is distributed under the terms of the Creative Commons Attribution Noncommercial License which permits any noncommercial use, distribution, and reproduction in any medium, provided the original author(s) and source are credited.

\section{References}

1. Soliman PT, Slomovitz BM, Broaddus RR et al (2004) Synchronous primary cancers of the endometrium and ovary: a single institution review of 84 cases. Gynecol Oncol 2004(94):456-462
2. Zaino R, Whitney C, Brady MF et al (2001) Simultaneously detected endometrial and ovarian carcinoma-a prospective clinicopathologic study of 74 cases: a Gynecologic Oncology Group study. Gynecol Oncol 83:355-362

3. Ramus SJ, Elmasry K, Luo Z et al (2008) Predicting clinical outcome in patients diagnosed with synchronous ovarian and endometrial cancer. Clin Cancer Res 14:5840-5548

4. Walsh C, Holschneider C, Hoang Y et al (2005) Coexisting ovarian malignancy in young women with endometrial cancer. Obstet Gynecol 106:693-699

5. Ulbright T, Roth L (1985) Metastatic and independent cancers of the endometrium and ovary: a clinicopathologic study of 34 cases. Hum Pathol 16:28-34

6. Pecorelli S (2009) Revised FIGO staging for carcinoma of the vulva, cervix, and endometrium. Int J Gynecol Obstet 105:103-104

7. Williams MG, Bandera EV, Demissie K et al (2009) Synchronous primary ovarian and endometrial cancers: a population-based assessment of survival. Obstet Gynecol 113:783-789

8. Castro IM, Connell PP, Waggoner S et al (2000) Synchronous ovarian and endometrial malignancies. Am J Clin Oncol 23:521525

9. Herrinton LJ, Voigt LF, Weiss NS et al (2001) Risk factors for synchronous primary endometrial and ovarian cancers. Ann Epidemiol 11:529-533

10. Signorelli M, Fruscio R, Lissoni AA et al (2008) Synchronous early-stage endometrial and ovarian cancer. Int J Gynecol Obstet 102:34-38

11. Lee NK, Cheung MK, Shin JY et al (2007) Prognostic factors for uterine cancer in reproductive-aged women. Obstet Gynecol 109:655-662

12. Gemer O, Bergman M, Segal S (2004) Ovarian metastasis in women with clinical stage I endometrial carcinoma. Acta Obstet Gynecol Scand 83:208-210

13. Wright JD, Buck AM, Shah M et al (2009) Safety of ovarian preservation in premenopausal women with endometrial cancer. J Clin Oncol 27:1214-1219

14. Navarria I, Usel M, Rapiti E et al (2009) Young patients with endometrial cancer: how many could be eligible for fertility-sparing treatment? Gynecol Oncol 114:448-451

15. Ben-arie A, Perlman S, Hazan Y et al (2004) High-risk endometrial cancer in young indigent women. Int $\mathrm{J}$ Gynecol Cancer 14:927-930

16. Tran BN, Connell PP, Waggoner S et al (2000) Characteristics and outcome of endometrial carcinoma patients age 45 years and younger. Am J Clin Oncol 23:476-480

17. Soliman PT, Oh JC, Schmeler KM et al (2005) Risk factors for young premenopausal women with endometrial cancer. Obstet Gynecol 105:575-560

18. Ota T, Yoshida M, Kimura M et al (2005) Clinicopathologic study of uterine endometrial carcinoma in young women aged 40 years and younger. Int J Gynecol Cancer 15:657-662

19. Kotera K, Moriyama S, Ishimaru T et al (2004) Wedge resection of the opposite ovary in conservative surgery of malignant ovarian tumor: is there any value? Nippon Rinsho 62:527-530

20. Zivanovic O, Carter J, Kauff ND et al (2009) A review of the challenges faced in the conservative treatment of young women with endometrial carcinoma and risk of ovarian cancer. Gynecol Oncol 115:504-509

21. Shamshirsaz AA, Withiam-Leitch M, Odunsi K et al (2007) Young patients with endometrial carcinoma selected for conservative treatment: a need for vigilance for synchronous ovarian carcinomas, case report and literature review. Gynecol Oncol 104:757760

22. Ramus SJ, Elmasry K, Luo Z et al (2008) Predicting clinical outcome in patients diagnosed with synchronous ovarian and endometrial cancer. Clin Cancer Res 14:5840-5848 